Rome III criteria for IBS-D. In Group 1 the prevalence of IBS and its differing subtypes (IBS-D, IBS-C, mixed IBS (IBS-M) and unspecified IBS) were determined using the Rome III Diagnostic Questionnaire. In groups 2 and 3 , demographic data and diagnostic yield of any investigations undertaken as part of the diagnostic workup were evaluated. Statistical analysis was performed using SPSS version 17.0 (SPSS Inc, Chicago, IL) with Fisher's exact test used to compare categorical data, and an unpaired T-test used to compare continuous data

Results IBS prevalence in healthy volunteers (Group 1) was $6 \%$ $(60 / 1002)$, with $80 \%$ being female $(p<0.0001)$. Mixed IBS was the most common IBS subtype (Table 1 ), with IBS-C patients being significantly older than other patients with IBS (mean age 45 vs 30 years, $p=0.027$ ). When comparing Groups 2 and 3 , patients with IBS-C underwent a total of 56 additional investigations (including radiological, endoscopic investigations, breath tests, SeHCAT scan, faecal pancreatic elastase), significantly lower than the number of investigations undertaken in the IBS-D group of $885(p<0.001)$. Whilst further investigations in Group 3 identified an alternative diagnosis to IBS-D in $25 \%$, the 56 additional tests undertaken in Group 2 did not help establish an alternative diagnosis to IBS-C in any of the patients.

Abstract PTU-134 Table 1 Prevalence rates of differing types of IBS in Group $1(\mathrm{n}=1002)$

\begin{tabular}{llllll}
\hline Subytype of IBS & $\begin{array}{l}\text { Number } \\
\text { of Patients }\end{array}$ & Prevalence (\%) & Sex (F:M) & Mean Age & $\begin{array}{l}\text { Standard } \\
\text { Deviation }\end{array}$ \\
\hline IBS-D & 14 & 1.4 & $7: 7$ & 32 & 18 \\
IBS-C & 7 & 0.7 & $6: 1$ & 45 & 21 \\
IBS-M & 27 & 2.7 & $24: 3$ & 32 & 15 \\
Unspecified IBS & 12 & 1.2 & $11: 1$ & 25 & 7 \\
\hline
\end{tabular}

Conclusion This is the first study to evaluate the population prevalence of differing IBS subtypes within a UK population. Whilst, further investigation of IBS-D patients may lead to an alternative diagnoses and instigation of an appropriate management strategy, the merits of further investigation in IBS-C patients is to be questioned.

Disclosure of Interest None Declared

\section{PTU-135 MECHANISM OF ACTION FOR LINACLOTIDE-INDUCED ABDOMINAL PAIN RELIEF}

doi:10.1136/gutjnl-2013-304907.225

1."S M Brierley, ' $\mathrm{J}$ Castro, 'A Harrington, ${ }^{1} \mathrm{P}$ Hughes, ${ }^{1} \mathrm{C}$ Martin, ${ }^{2} \mathrm{~A}$ Silos-Santiago, ${ }^{2} \mathrm{C}$ Kurtz, ${ }^{3} \mathrm{~A}$ Blackshaw. 'Medicine, University of Adelaide, Adelaide, Australia; 2Ironwood Pharmaceuticals, Cambridge, United States; ${ }^{3}$ Wingate Institute for Neurogastroenterology, London, UK

Introduction Cyclic GMP (cGMP) is a 2nd messenger produced in intestinal epithelial cells in response to guanylate cyclase $C$ receptor (GCC) activation. Linaclotide (LIN), an investigational GCC agonist (GCCA), improved constipation and reduced abdominal pain in patients with irritable bowel syndrome with constipation (IBS-C) in clinical trials. We have shown that exogenous extracellular cGMP has contrasting effects on colorectal (CR) afferent mechanosensitivity. ${ }^{1}$ Here we assessed the effects of GCCAs on CR afferent mechanosensitivity in healthy and chronic visceral hypersensitivity (CVH) mouse models

Methods We investigated CVH in healthy mice and CVH 28 days post-TNBS administration, when inflammation had resolved and nociceptors were mechanically hypersensitive. Mechanosensory responses of CR splanchnic nociceptors and pelvic mucosal afferents were compared in vitro \pm GCCAs STc $(1,50,250,1000 \mathrm{nM})$ and LIN (1, 30, 100, 300, 1000nM), which were applied individually to the CR mucosal surface. GCC expression in the CR mucosa was determined via qRT-PCR
Results In healthy mice, STc dose-dependently (50, 250, 1000nM) reduced nociceptor mechanosensitivity (max. effect at $1000 \mathrm{nM}$ $[n=10],-38 \% ; p<0.001)$. This effect was more potent in $\mathrm{CVH}$, with various doses of STc $(1,50,250,1000 \mathrm{nM})$ all significantly reducing mechanosensitivity (max. effect at $1000 \mathrm{nM}[\mathrm{n}=10]$, $-53 \% ; p<0.001$ ). In healthy mice, LIN significantly reduced nociceptor mechanosensitivity at doses of $300 \mathrm{nM}$ and $1000 \mathrm{nM}$ (max. effect at $1000 \mathrm{nM}[\mathrm{n}=7],-48 \%$; $\mathrm{p}<0.01)$. In $\mathrm{CVH}$ this effect was dose-dependent and more potent, with LIN (100, 300, $1000 \mathrm{nM})$ significantly reducing nociceptor mechanosensitivity (max. effect at $1000 \mathrm{nM}[\mathrm{n}=5],-59 \% ; \mathrm{p}<0.001)$. By contrast, in pelvic nerves STc increased low-threshold pelvic mucosal afferent mechanosensitivity in healthy mice ( $\mathrm{n}=7 ; \mathrm{p}<0.001)$, an effect completely lost in $\mathrm{CVH}$ $(n=7 ; p>0.05)$. qRT-PCR analysis revealed abundant GCC expression in CR mucosa of both healthy and CVH mice

Conclusion STc and LIN significantly reduced colonic nociceptor mechanosensitivity, with greatest effect in $\mathrm{CVH}$. Although these overall effects mirror those of exogenously applied cGMP, GCCAs are more potent at inhibiting nociceptors. Overall, LIN induced the greatest inhibitory effects on nociceptors, particularly in $\mathrm{CVH}$. Reducing colonic nociceptive input would help to reduce pain, which supports LIN clinical data showing a significant reduction in abdominal pain in humans with IBS-C. Increased mucosal afferent sensitivity may help coordinate defecation.

Disclosure of Interest S. Brierley Grant/Research Support from: Ironwood Pharmaceuticals, J. Castro: None Declared, A. Harrington: None Declared, P. Hughes: None Declared, C. Martin: None Declared, A. Silos-Santiago Employee of: Ironwood Pharmaceuticals, C. Kurtz Employee of: Ironwood Pharmaceuticals, A. Blackshaw Grant/ Research Support from: Ironwood Pharmaceuticals.

\section{REFERENCE}

1. Gastro. 2011. Supported by Ironwood Pharmaceuticals Inc and by Forest Laboratories Inc. Medical writing assistance was provided by Complete Medical Communications, funded by Almirall.

\section{PTU-136 PREVALENCE OF FUNCTIONAL GASTROINTESTINAL DISORDERS IN CONSECUTIVE NEW PATIENT REFERRALS TO A GASTROENTEROLOGY CLINIC}

doi:10.1136/gutjnl-2013-304907.226

1.*U Shivaji, 'D Gracie, 'A Sainsbury, 'A C Ford. 'Gastroenterology, Leeds Teaching Hospitals, Leeds, UK

Introduction Functional gastrointestinal disorders (FGIDs), such as irritable bowel syndrome (IBS) and functional dyspepsia (FD), are common in the community. However, the amount of time that a Gastroenterologist spends dealing with these conditions in the outpatient department has not been well-described. We aimed to examine this issue.

Methods Review of consecutive unselected new patient referrals to a single Gastroenterologist's outpatient clinic during a 2-year period, from January 2010 to December 2011. All clinic letters were reviewed retrospectively, and symptoms reported by the patient at the initial consultation were recorded. Radiology, endoscopy, chemical pathology, and histopathology databases were then cross-examined in order to ascertain the final diagnosis following full investigation, to the level deemed appropriate by the consulting physician.

Results There were 397 consecutive unselected new patient referrals (mean age 53.2 years, 231 (58.2\%) female) to a single Gastroenterologist between January 2010 and December 2011. Of these, 155 $(39.0 \%)$ were judged as being entirely due to an FGID after investigation, and another $12(3.0 \%)$ were deemed as being partially related to FGIDs, meaning that 167 (42.0\%) patients reported symptoms compatible with an FGID. The final diagnoses are listed in Table 1, with some patients reporting symptoms consistent with 